\title{
Acute Ischemic Stroke as the Initial Presentation of Takayasu Arteritis in a 16-Year-Old
}

\section{INTRODUCTION}

Takayasu arteritis (TA) is a rare chronic disease of unknown etiology that affects the aorta and large vessels. The development of neurological symptoms as the initial presentation of TA is rare, and when they are present, usually the most common cause of these symptoms are related to extracranial vascular abnormalities. Intracranial manifestations of TA are less frequent and ischemic stroke as the presentation of this disease is rarely encountered. Although TA is an uncommon cause of acute ischemic stroke, it should be kept in mind, especially in the young.

\section{CASE PRESENTATION}

We present a case of a16-year-old girl with an unremarkable past medical history that was admitted to our emergency room with a 48-hour severe rightsided hemiparesis and aphasia. Magnetic resonance and angiography (MRA) of the neck and the brain was performed revealing an subacute left middle cerebral artery (MCA) ischemic stroke due to total occlusion of the left internal carotid artery (ICA) and its branches (Fig.1 and 3). The neck MRA showed total occlusion of the left common carotid artery (CCA) and its branches, and also proximal severe stenosis of the right CCA, left subclavian and both vertebral arteries (Fig. 2). The patient received corticoid therapy and endovascular treatment with a carotid wall stent (Fig. 4) and was discharged a week later with minimal right-sided hemiparesis.

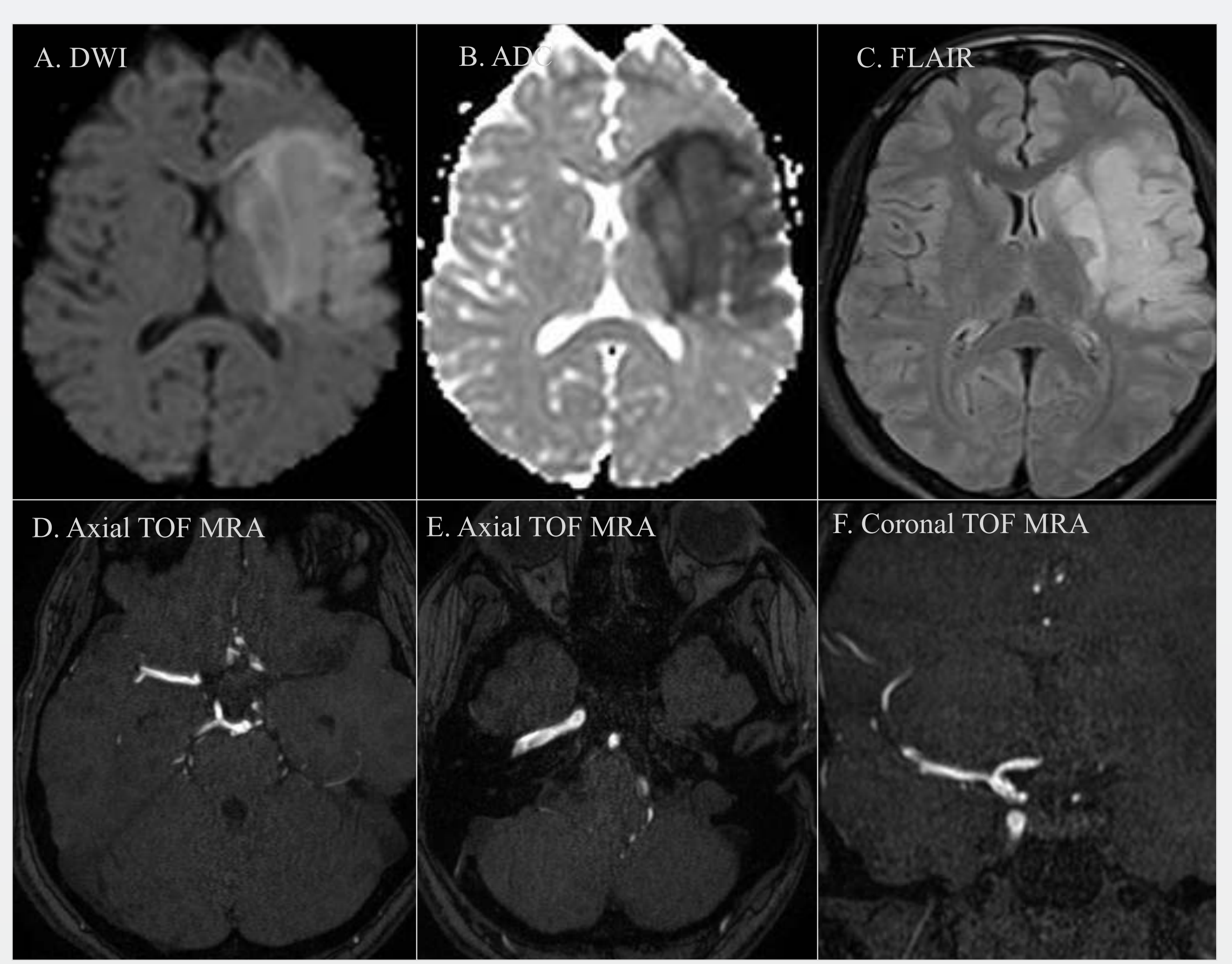

Figure 1: DWI and $\mathrm{ADC}(\mathrm{A}, \mathrm{B})$ images show an area of restricted diffusion in the left middle cerebral artery territory. FLAIR MR image (C) shows a corresponding area of high signal intensity in relation with an early subacute stroke. TOF-ARM images (D, E, F) show total occlusion of the left internal carotid artery and middle cerebral artery.

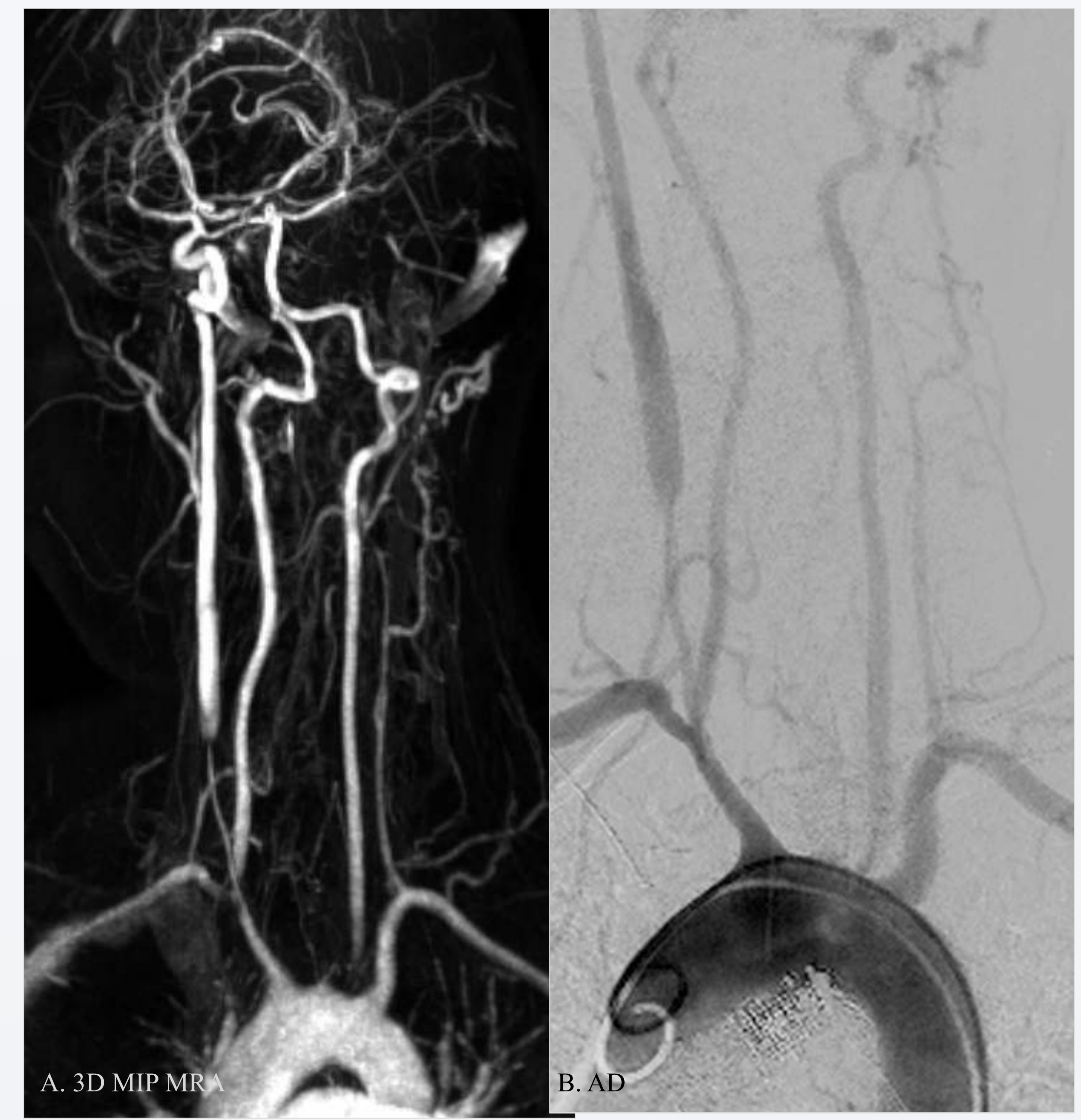

Figure 2: 3D MIP MRA of brain and neck and (A) AD images show total occlusion of the left common carotid artery and its branches. There is also proximal severe stenosis of the right common carotid artery and both vertebral arteries.

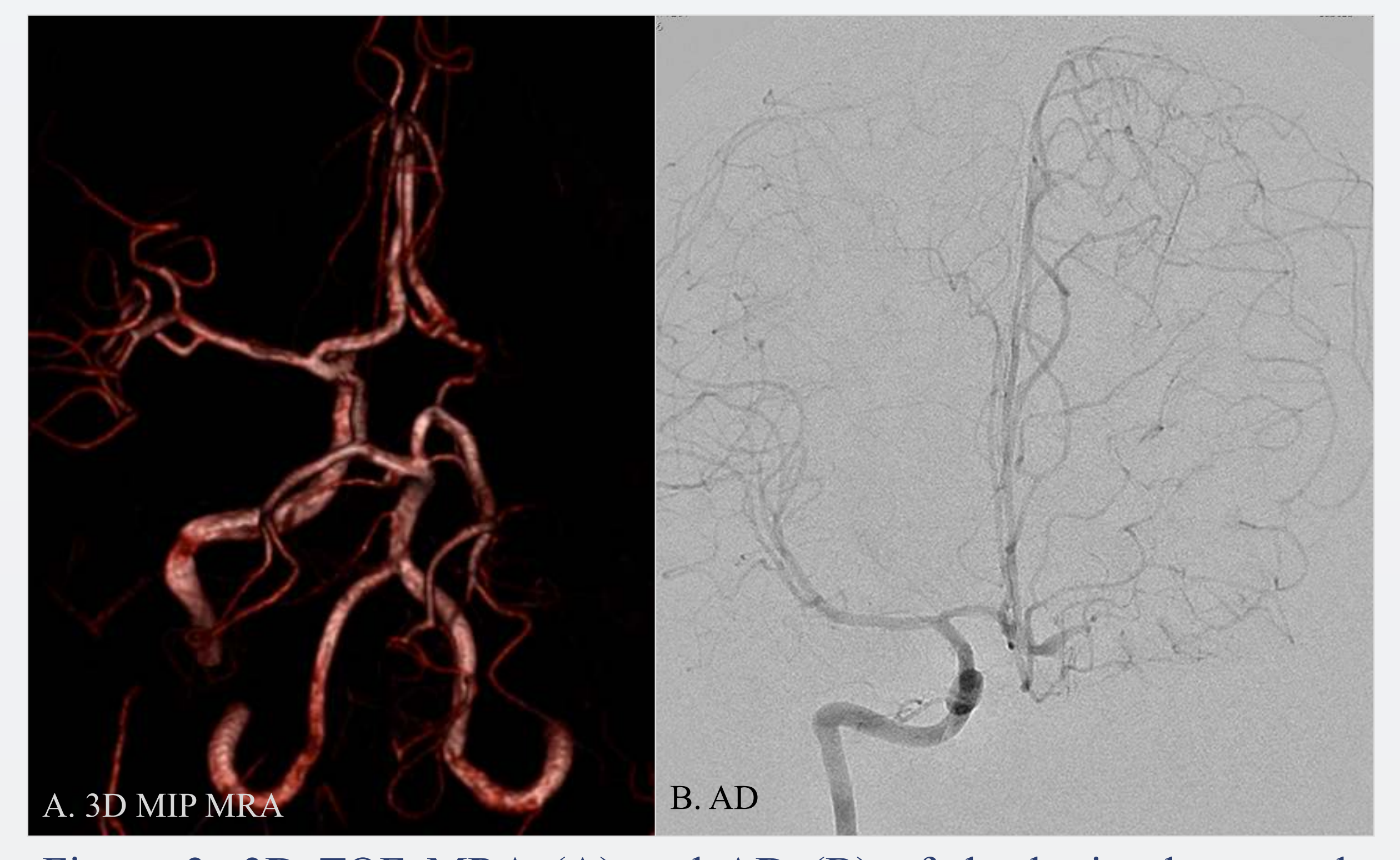

Figure 3: 3D TOF MRA (A) and AD (B) of the brain show tota occlusion of the left internal carotid artery and its branches.

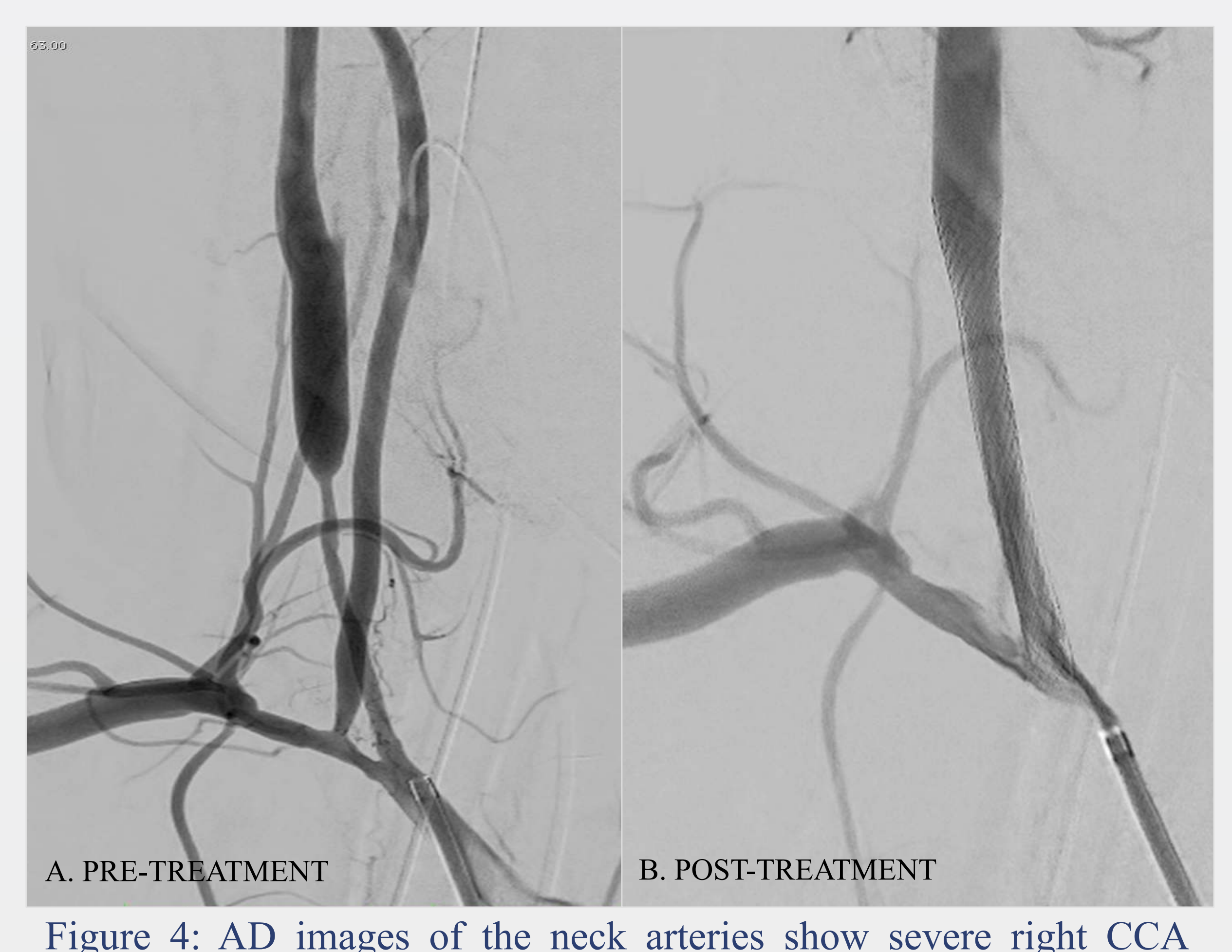

steneosis (A). The patient was treated with carotid wall stent (B)

\section{DISCUSSION}

Takayasu arteritis is a chronic vasculitis of unknown etiology that mainly involves the aorta and its major branches. It affects mainly women and is more prevalent in Asia and Latino-American countries. The clinical symptoms are variable and range from asymptomatic cases to arterial claudication and endorgan ischemia depending on location and severity of the lesions. The involved arteries suffer of granulomatous inflammation that leads to wall thickening, stenosis and occlusion. Aneurysmal dilatation and arterial dissections can be seen, although they are uncommon findings.

Neurological manifestations without systemic symptoms in TA are rare and if present, they are usually secondary to involvement of extracranial vessels. Extracranial vascular abnormalities are the most common cause of ischemic neurologic symptoms in TA with an incidence of $21-65 \%$ compared to the incidence of intracranial involvement in TA with only $10-20 \%$.

The underlying mechanisms of ischemic strokes in TA remain unclear and various have been described, including, vascular occlusion secondary to arterial thrombosis and vasculitis, carotid aneurysm, embolism, hemodynamic secondary to stenosis or vascular steal, vasospasm, and dissection.

Imaging diagnosis of intracranial TA includes CTA, MRA and DSA as the gold standard method. Brain CT and MRI are also performed for parenchyma evaluation in the presence of acute and chronic infarcts, microhemorrhage and white matter disease.

\section{CONCLUSION}

Although ischemic stroke as the initial manifestation of Takayasu arteritis is uncommon, it should be considered as a differential diagnosis, especially in the young. Ischemic stroke is associated with high morbidity and early detection and treatment is essential in order to prevent progression of the disease and neurological disabilities.

\section{REFERENCES}

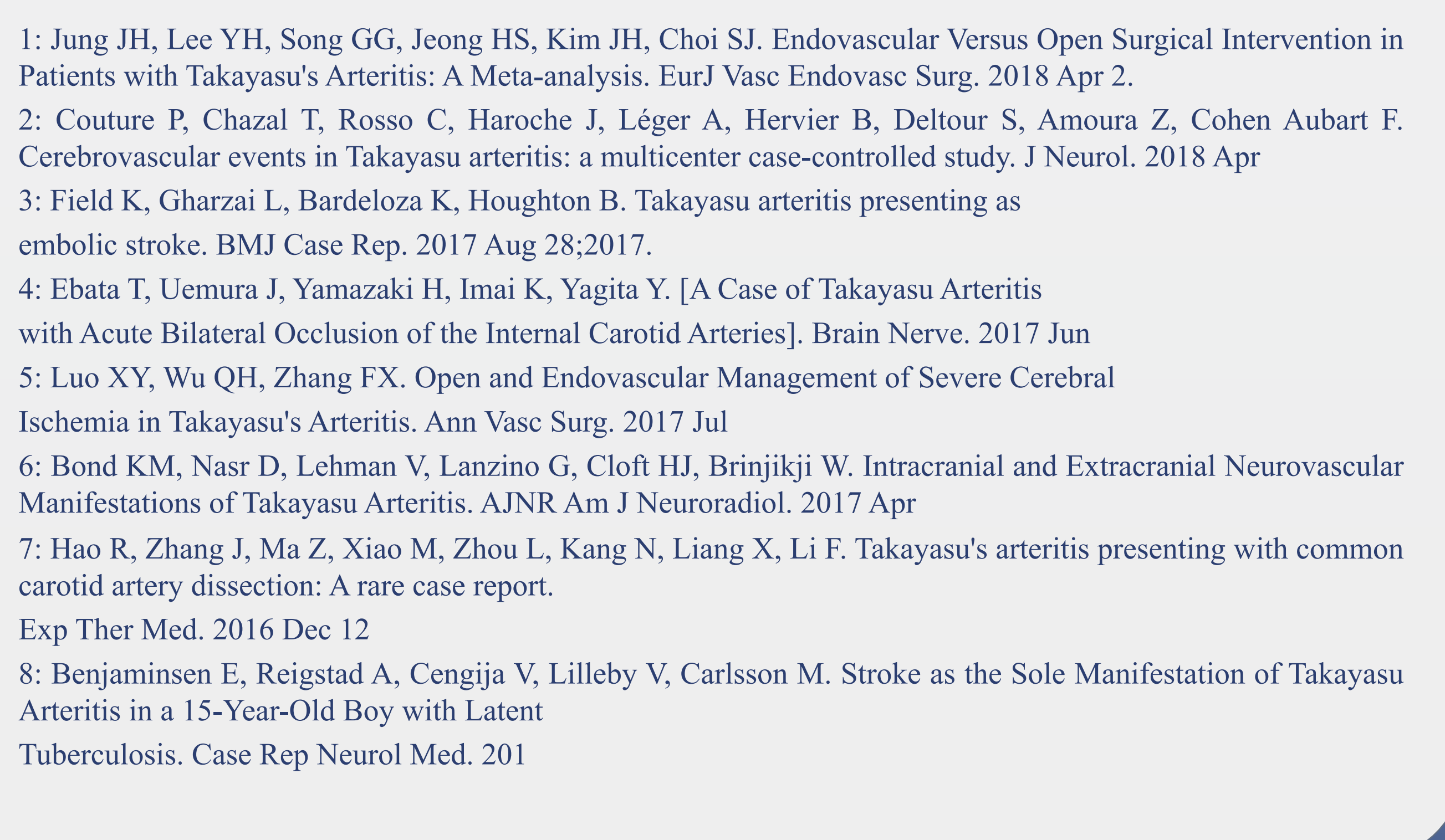

